\title{
Les problématiques contemporaines du pardon au miroir du massacre des harkis
}

\section{Emmanuel Brillet}

\section{(2) OpenEdition}

1 Journals

\section{Édition électronique}

URL : http://journals.openedition.org/conflits/404

DOI : $10.4000 /$ conflits.404

ISSN : $1777-5345$

Éditeur :

CCLS - Centre d'études sur les conflits lilberté et sécurité, L'Harmattan

Édition imprimée

Date de publication : 4 mars 2001

ISBN : 2-7475-0725-4

ISSN : 1157-996X

\section{Référence électronique}

Emmanuel Brillet, « Les problématiques contemporaines du pardon au miroir du massacre des harkis ", Cultures \& Conflits [En ligne], 41 | printemps 2001, mis en ligne le 14 septembre 2006, consulté le 30 mars 2021. URL : http://journals.openedition.org/conflits/404 ; DOI : https://doi.org/10.4000/ conflits. 404

Ce document a été généré automatiquement le 30 mars 2021.

Creative Commons License 


\title{
Les problématiques contemporaines du pardon au miroir du massacre des harkis
}

\author{
Emmanuel Brillet
}

1 Les problématiques contemporaines du pardon au miroir du massacre des harkis Emmanuel BRILLET

2 Fil d'Ariane des relations franco-algériennes depuis maintenant près de quarante ans, la question de la «normalisation» des relations diplomatiques entre les deux protagonistes d'une guerre restée longtemps innommée du côté français, ou mythifiée du côté algérien, affleure celle, autrement plus sensible, de la réconciliation entre « isolats mémoriels, [ces] mémoires particulières en lutte les unes contre les autres qui sont souvent celles des traumatisés $»^{1}$. À cet égard, plus encore que le débat entourant la réminiscence tardive des faits de torture pendant la guerre d'Algérie, c'est son orchestration médiatique qui est ici significative. Lutte frontale entre deux mémoires particulières (celle, collégiale, des intellectuels en guerre d'Algérie et celle, institutionnelle, de l'armée), cette réactualisation soudaine d'une polémique déjà ancienne ne fait qu'accroître le sentiment d'émiettement des requêtes mémorielles. Ainsi que le souligne fort justement Paul Thibaud, si l'équation guerre d'Algérie = torture venait à prévaloir dans l'opinion, c'est un autre moment essentiel pour comprendre la guerre d'Algérie qui pourrait être éludé, soit «l'échec des accords d'Évian dans une conjonction d'événements sinistres : O.A.S., harkis, exode des piedsnoirs, mise en place par la force d'un pouvoir algérien militaro-populiste $»^{2}$. Dès lors, la prévalence médiatique de la geste protestataire, loin de «désenclaver les mémoires traumatisées $»^{3}$, ne ferait que nourrir la frustration ou l'acrimonie souterraines d'autres mémoires particulières. Nous entendons précisément ici rendre sa part à la demande de reconnaissance de la communauté harkie, laquelle vit son rapport au passé dans un contexte d'isolement moral et d'incuriosité intellectuelle qui l'expose, plus que d'autres protagonistes de la première guerre d'Algérie, aux assignations statutaires ou aux analyses réductrices ${ }^{4}$. Pour autant, notre optique, en accord avec la thématique 
générale de ce numéro, n'est pas d'entrer dans le détail des événements historiques mais d'étayer l'étude des problématiques contemporaines du pardon sur le cas d'espèce que représente le contentieux historique entre la communauté harkie et l'État algérien d'une part, entre la communauté harkie et l'État français d'autre part ${ }^{5}$. A cet égard, nous verrons que l'analyse des aspects rhétoriques du différend tout autant que celle des enjeux éthiques et pratiques du pardon trouvent, dans le contexte de la seconde guerre d'Algérie, des prolongements inattendus et éclairants. Ainsi en va-t-il de la convocation abusive de la thématique harkie à des fins attentatoires mais aussi, s'agissant de l'ingénierie politique du pardon, du contre-exemple paradigmatique de la loi de « concorde civile " ${ }^{6}$. Chacun s'accorde, d'une rive à l'autre de la Méditerranée, à considérer comme formellement tragique la destinée - depuis l'engagement jusqu'à la mort ou l'exil - de ceux que l'on désigne sous le terme générique de harkis. Pour autant, l'examen des attendus du drame, et de ses conséquences, semble ne pas pouvoir ou ne pas devoir avoir lieu : nul espace de délibération politique où puissent débattre de manière contradictoire les protagonistes du drame, nul espace d'intelligibilité où puisse s'exprimer publiquement le dissensus ${ }^{7}$. Dès lors, comment faire sourdre ce qui, dans la ressouvenance du règlement politique de la guerre d'Algérie, et, plus encore, de ses conséquences humaines, fait écran lorsqu'il s'agit de se définir collectivement ${ }^{8}$ par rapport à cette période en général, par rapport à cet épisode en particulier ? Ce qui fait écran, d'abord, est «ce point d'étrange résignation qui, en Algérie, est toujours déjà atteint", "[cette] mortification massive qui, par spasmes aigus, dégage sa dose d'horreur, puis se replie dans le banal et le quotidien $»^{9}$. Plus que tout autre mode d'expression et d'objectivation du politique, c'est la violence qui, en effet, semble le mieux caractériser la dynamique des relations sociales dans et à l'extérieur de la sphère du pouvoir en Algérie depuis l'indépendance. Le point de savoir si cette violence est structurelle, c'est-à-dire structurellement liée à l'histogenèse de la sortie hors de la situation coloniale, fait débat. A n'en pas douter, cependant, par-delà même le repli sur soi en forme de table rase engagé par le F.L.N. au moment de l'exode forcé des Algériens de confession juive et catholique, le massacre des harkis à l'été et à l'automne 1962 participe de ces souvenirs archaïques qui servent de "loi narcissique » au corps social algérien. Car dès lors que ce "blocage [cette blessure ?] narcissique » sert de "Loi du père " dans la geste nationaliste de l'Algérie post-coloniale, qui oserait demander à se libérer de « cette sorte de résignation à ce que la scène, celle du sacrifice humain, enfouie dans l'archaïque, soit ainsi représentée, passée à l'acte $»^{10}$ ? En Algérie, la valorisation de l'identité en tant que source tautologique et totalitaire de légitimation politique prévaut sur les modes démocratiques d'élection du personnel politique et sur le libre débat d'idées. Récits et interprétations historiques servent à beaucoup d'autres choses qu'à l'avancée de la connaissance, et le refus d'évoquer le massacre des harkis autrement que sur le mode de "l'implicite castrateur $»^{11}$ exprime le refus de modifier une identité que structure la geste officielle de la guerre d'Algérie. Car pour violentes que fussent les rivalités internes au cénacle algérien, les élites au pouvoir depuis 1962 ont ceci en commun qu'elles entendent capitaliser à des fins politiques, et quoi qu'il en coûte à l'avènement d'une société ouverte, la légitimité que leur confère leur participation à la guerre de libération nationale. Le récit mythifié des origines tendant à faire accroire à l'unanimité des masses derrière le F.L.N., aussi bien que la stigmatisation générique de la figure du harki en tant que figure de «l'ennemi intérieur ", sont autant de procédés dilatoires qui, postulant la monovalence des aspirations du corps social algérien, permettent d'imputer les ferments de division 
interne à d'autres que soi ${ }^{12}$. Dès lors, accepter de demander pardon aux harkis, n'est-ce pas, pour les autorités algériennes, risquer d'altérer les frontières de l'identité postcoloniale et, par-là même, faire vaciller le socle symbolique sur lequel s'étayent les pratiques monolithiques du "système» depuis l'indépendance? Ce qui fait écran, encore, est ce point d'étrange résignation qui, en France, semble toujours déjà atteint lorsqu'il s'agit d'envisager les conséquences humaines de la politique de « dégagement » entreprise en Algérie par le pouvoir gaullien. À Patrick Poivre d'Arvor, qui, à l'occasion de la traditionnelle garden-party de l'Elysée, le 14 juillet 2000, lui demanda ce qu'il pensait du parallèle entre harkis et collaborationnistes établi publiquement par Abdelaziz Bouteflika au cours de sa récente visite d'État en France, Jacques Chirac tint le langage convenu et dilatoire du responsable politique mis dans l'embarras. S'il se déclara choqué par les déclarations du chef de l'État algérien, il n'expliqua pas pourquoi ce parallèle était infamant. De la même manière, éludant la question de la responsabilité de l'État français dans l'abandon à un sort prévisible de la majeure partie de ceux qui avaient pris une part active à l'administration (notables, fonctionnaires) et à la défense (supplétifs) de l'Algérie coloniale. Navigant à vue, le chef de l'État se contenta de rappeler que les anciens harkis et leurs enfants étaient des Français à part entière, bénéficiaires des mêmes droits et astreints aux mêmes devoirs que tous leurs concitoyens. Regard générique, regard méthodique, la geste officielle française de la guerre d'Algérie participe de l'expurgation embarrassée de toute résonance affective et, pour tout dire, de la censure d'un sentiment d'incomplétude hérité d'un conflit qui «n'est tout simplement pas mémorable, [qui] ne figure pas, après l'affaire Dreyfus et la Résistance, parmi les épopées du sens, les émergences de la justice à quoi les Français aiment à se référer $»^{13}$. "Si la mémoire [de la guerre d'Algérie] est en effet difficile, ajoute Paul Thibaud, c'est parce qu'il n'y a pas de lieu où elle pourrait s'ancrer, qu'il manque au drame une issue vraiment acceptable et vraiment compréhensible $»^{14}$. Ce qui fait écran, enfin, est la frontière symbolique de la correction ou de l'incorrection politique, qui explique en partie au moins l'inappétence des leaders d'opinion et des analystes pour cette question. Est en cause, plus précisément, l'étiquette "Algérie française $»^{15}$ accolée à la population harkie, marginalisant la prise en compte des questionnements afférents à sa destinée à quelques cercles restreints, eux-mêmes ostracisés; l'on pense notamment à Radio Courtoisie, qui ne cache pas ses accointances avec l'extrême droite. Là réside une différence essentielle avec le récent réinvestissement médiatique du débat relatif aux faits de torture pendant la guerre d'Algérie. De fait, ce débat, déjà très vif en son temps, est porté par toute une frange autrefois protestataire (voire révolutionnaire) de la gauche intellectuelle qui, aujourd'hui plus qu'hier, détient les clefs du savoir et contrôle les outils de sa diffusion. Autrement dit, le débat moral est aussi affaire de fenêtre d'opportunité, et toutes les mémoires traumatisées n'ont pas des chances égales de voir satisfaite leur demande de reconnaissance. Dès lors, comment faire en sorte que les choses se présentent autrement dans les rapports entre la communauté harkie et la société algérienne d'une part, entre la communauté harkie et la société française d'autre part, que comme choc entre des usages de la mémoire qui ont des fonctions identitaires symétriques, et empêchent symétriquement toute réflexion sur les responsabilités historiques? Au regard même des victimes, si la mémoire du massacre ${ }^{16}$ structure le lien social qui fait des anciens harkis et de leurs enfants une communauté, le pardon devient, à la limite, une menace de dissolution. Car toute reconnaissance, et a fortiori celle que confère le pardon, est à la fois un soulagement et une petite mort pour 
une communauté marquée par l'épreuve du désastre. Ce qui est proprement tragique, en définitive, dans la trame de la disparition des harkis, c'est "l'étroitesse de l'angle d'engagement des protagonistes $\aleph^{17}$, le fait aussi "qu'à travers la finitude de leurs points de vue chacun d'eux développe une visée de légitimation infinie $»^{18}$. La dispersion du sens apparait d'autant plus irréversible que le souvenir est recalcifié au gré des usages et mésusages de la mémoire. Le conflit ne porte pas seulement sur l'interprétation des faits, mais leur ordonnancement même est fragmenté, recomposé. Ainsi, écrit Olivier Abel, «le tragique de l'histoire oppose des mémoires incompatibles, non seulement parce que l'histoire est racontée dans une pluralité de récits formés à partir de points de vue séparés (...), mais souvent la temporalité commune est ellemême brisée, parce qu'il n'y a pas de question commune qui nous rendrait contemporains les uns des autres, il n'y a plus d'échange possible sous un principe commun ${ }^{19}$. L'incommensurabilité des points de vue, l'irréductibilité des temporalités narratives autour desquelles ces points de vue s'organisent, sont illustrées, en France, par la polémique née autour de la célébration par certaines autorités officielles de la date anniversaire de la proclamation du cessez-le-feu en Algérie le 19 mars 1962. De telles cérémonies commémoratives, célébrées à l'initiative de la F.N.A.C.A. ${ }^{20}$ et portant mention : «19-mars-1962 : fin de la guerre d'Algérie », sont conçues par l'ensemble des autres associations d'anciens combattants et de rapatriés comme une insulte à l'égard de la mémoire des centaines de militaires, des milliers de pieds-noirs et des dizaines de milliers de harkis et membres de leurs familles morts ou disparus après cette date ${ }^{21}$. À ce stade, «l'échange des mémoires est impossible parce que les mémoires sont enracinées dans un immémorial inaccessible à l'échange. Il y a une corporéité des identités historiques qui les rend incapables à partir d'un certain point de rendre raison d'elles-mêmes, qui les empêche de répondre à toutes les questions. Comme si l'identité était précédée par une dette transcendantale à tous les échanges qui l'avaient définie, ou par un oubli plus vaste et plus vivant que toutes ses remembrances $»^{22}$. Prenant acte de l'incommensurabilité des univers de justification dans le tragique de l'histoire, «la sagesse du pardon est une sagesse pratique qui ne propose pas une délivrance du tragique mais une délivrance dans le tragique même: le pardon est la vertu du compromis parce qu'il accepte le différend $»^{23}$. Non pas ce pardon moral élémentaire qui «rythme la mémoire ordinaire de l'échange, avec ses dettes rétribuables, effaçables", qui "suppose une temporalité causale et continue, où les maux ont des causes assignables quelque part dans la structure de l'échange », et qui "s'applique seulement à des situations claires, ou clarifiées par convention $»^{24}$; mais un

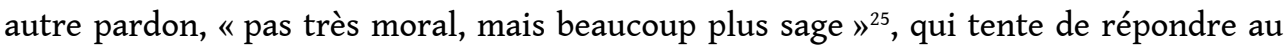
tragique de l'irréversible, qui touche à "des identités engoncées dans des conflits insurmontables, et qui disparaîtraient hors de ces conflits, à des mémoires enracinées dans des créances et des dettes impayables, comme enfouies dans un passé irréparable $»^{26}$. Ce pardon-là, « loin d'être une petite affaire de morale ou de religion personnelle, se tient partout où il y a un rapport au passé, à l'irréparable, à la mémoire, à une histoire qui n'est pas seulement celle des gloires mais aussi celle des souffrances $»^{27}$. Il s'agira, en l'espèce, d'ouvrir un débat éthique et pratique à propos du pardon, de ses règles et de ses conditions, afin d'envisager les moyens de "sortir de l'infernale antinomie entre la dette et l'amnésie $»^{28}$. Pour une définition opératoire du pardon en politique Nous empruntons ces quelques réflexions liminaires au philosophe Olivier Abel qui, au travers de différentes contributions théoriques et pratiques sur la manière d'aborder la question du pardon "là où l'histoire touche au tragique ", a 
participé de l'ouverture de ce débat - traditionnellement d'essence moraliste, philosophique et / ou théologique - aux sciences sociales: histoire, sociologie, politologie, psychanalyse ${ }^{29}$. Notre optique, qui participe de ce souci de pluraliser et de « pragmatiser » les axes de réflexion attenants à la question du pardon, vise à proposer une définition opératoire de cette notion, susceptible de «coller » aux problématiques socio-politiques contemporaines :

- soit, d'une part, une définition du pardon qui soit suffisamment lâche et pragmatique pour ne pas risquer de trahir la complexité et la singularité des situations historiques. Il ne s'agit pas de proposer une définition "idéale», esthétique du pardon, mais simplement d'en dégager l'ossature minimale, le socle, soit les conditions de possibilité éthiques et pratiques à l'échelle de nos sociétés ;

- soit, d'autre part, une définition du pardon suffisamment assurée d'elle-même pour satisfaire aux conditions de réciprocité dans l'échange sans pour autant relativiser la part de responsabilité de l'agresseur. Il ne s'agit pas forcément de s'entendre sur les faits, comme si l'agresseur et la victime pouvaient soudainement jeter un regard extérieur et convergent sur les faits dont ils ont été les protagonistes. Mais il s'agit d'accepter l'autre version des faits, d'accepter l'échange des témoignages, en somme, de recontextualiser l'objet du différend. Concurremment, et plus particulièrement dans le cas des massacres collectifs, il y a une forme de hiérarchisation des responsabilités à établir. Car une violence meurtrière a été perpétrée qui, précisément parce qu'elle s'est évertuée à revêtir un caractère inexpiable ${ }^{30}$, demande à être reconnue par l'agresseur pour ce qu'elle est, ce quelles que fussent les justifications circonstancielles dont cette violence a été entourée. Si l'on s'en tient à l'appréhension conceptuelle de la dette et de l'amnésie, par-delà même l'étude de cas qui nous intéresse, ces deux notions, « avec les types de rapport à l'histoire qu'elles supposent, appartiennent à la même problématique où la mémoire est de toute façon tronquée $»^{31}$. Abstraction faite de la matière à laquelle elles s'appliquent, elles participent d'une forme d'homologie structurale, d'une même logique de l'enfermement ou du renfermement. Toutefois, si l'on envisage la dette et l'amnésie dans leur inscription et dans leur confrontation pratiques, si l'on se place sur le plan sensible du vécu des acteurs en situation, nous ne saurions considérer les configurations d'acteurs qu'elles décrivent comme participant de dynamiques sociales et relationnelles convergentes, tant en termes de contraintes que de devoirs ou d'obligations. On conçoit sans peine, en effet, que la structure de l'échange sous-jacente à l'inscription pratique de la logique de la dette et de la logique de l'amnésie est discontinue, asymétrique, et que le rapport des forces y est inégal. Tandis que l'une (la logique de la dette) est subie et ressortit du poids de l'histoire, l'autre (la logique de l'amnésie) est « agie » et ressortit du choix de l'histoire. La logique de la dette participe de la cristallisation de la mémoire du tort chez la victime. Elle revêt un caractère obsessionnel qui s'objective sur le mode du ressentiment et de la fixation. Elle est une forme - une forme au long cours - de la sidération : « Il y a un point à partir duquel la dette n'est plus qu'une obsession, comme l'oubli n'était qu'amnésie. La dette fait alors que l'on réagit à tout comme s'il s'agissait toujours de la même chose, que tout réactive. Elle rend incapable de réagir à autre chose; elle rend incapable d'agir, simplement, à nouveau (...). Dans la logique de la dette apparaît une mémoire malade, incapable d'oublier ni d'effacer, et donc incapable de se souvenir d'autre chose $»^{32}$. À l'inverse, la logique de l'amnésie volontaire, de l'oubli «agi» participe chez l'offenseur de l'euphémisation de la mémoire de la faute, de sa réorchestration. Elle revêt, à ce titre, le caractère d'une geste. Dès lors, le pardon, s'il se 
donne pour objet de "briser la dette et [de] briser l'oubli », comme nous y invite Olivier Abel, ne saurait dans les faits mobiliser uniment la victime et son offenseur. De fait, l'acte de pardon, à travers lequel la victime dépare la mémoire du tort de son caractère obsessionnel et se libère du ressentiment qu'elle nourrissait à l'égard de son agresseur, ne saurait avoir de sens s'il n'est précédé par l'acte de reconnaissance de la réalité et de la gravité des faits à travers lequel l'offenseur rencontre et rend compte de sa faute ; à travers lequel, surtout, il accepte de restaurer la réciprocité dans ses relations avec la victime. Autrement dit, l'acte de reconnaissance est un pré-requis, une condition sine qua non de l'acte de pardon. Là où l'histoire touche au tragique, le pardon ne saurait rétablir la réciprocité dans les termes de l'échange si, en dernière instance, il concevait cet impératif de réciprocité comme pouvant s'affranchir de l'exploration des faits et de leurs effets ${ }^{33}$, et, par là-même, de la responsabilité des acteurs. Comment concevoir, comment avaliser une politique du pardon si, réduite à l'état de simple formule de politesse, elle méprisait la réalité et la gravité des faits au point de tenir pour négligeable la responsabilité des acteurs? N'est-ce pas là la définition même du pharisaïsme? Si elle participe d'un acte formel ou d'une décision unilatérale, la demande de pardon ne saurait être qu'un état de fait arbitraire, sans portée ni résonance, un chapitre que l'on clôt, en somme, quand tout devrait être revisité, "publicisé ", débattu. Elle trahit alors la vocation première du pardon qui est de rétablir la réciprocité dans la relation à l'autre, de restaurer de l'intelligibilité et de la fluidité dans le rapport au passé et dans les rapports humains; il ne s'agit pas de «liquider» le contentieux, mais de le dénouer, de le rendre sinon soluble, du moins intelligible, à même de donner prise à un débat contradictoire : "Il s'agit [pour un corps endeuillé ou dans l'impasse] de se pluraliser, pour que s'ouvre un jeu de la vie où la rencontre et le conflit soient possibles sans qu'on en meure. (...) Pour se rencontrer il faut être "contre", différent, mais assez serein dans cette différence pour ne pas croire que l'enjeu de la rencontre c'est que l'un ou l'autre doit rester sur le carreau (...) ; pour rendre enfin possible des luttes qui ne soient pas des meurtres; pour permettre d'autres violences que narcissiques $\aleph^{34}$. Une demande de pardon unilatérale de l'agresseur à la victime, comme une sorte de mise en demeure, s'apparente à un nouvel acte sinon d'agression, du moins de domination, comme si, lors même qu'il s'agirait d'offrir réparation à ceux que l'on a offensés, il revenait encore et toujours à l'agresseur de poser ses conditions ; comme si, à l'instar de l'acte d'agression et / ou de stigmatisation, l'acte même de réparation devait être suspendu à l'initiative de l'offenseur. La demande de pardon, dans ces conditions, ne revêt que trop le caractère d'une fuite des responsabilités, ne s'apparente que trop à une échappatoire, comme s'il suffisait de demander pardon pour être pardonné tout en faisant l'économie d'une anamnèse véritable. À son tour, le pardon accordé par la victime à son agresseur, s'il résulte d'une initiative unilatérale de la victime, lors même que l'agresseur n'aurait fait montre d'aucune velléité de reconnaissance de la réalité et de la gravité des faits, lors même - a fortiori - qu'il n'aurait fait montre d'aucun signe de repentance, ne s'apparente-t-il pas à une forme de renoncement, comme si la sérénité, la tranquillité d'esprit pouvaient se gagner au prix de la dignité, la concorde au prix de la justice ? La victime peut-elle ou doit-elle attendre de l'offenseur que, par la grâce, par la magie d'un pardon non sollicité, il se départisse de tout mépris, de toute morgue, qu'il abandonne toute velléité de domination? N'est-il pas illusoire, sinon absurde, d'attendre de l'agresseur qu'il vous rende grâce d'être pardonné pour des faits dont il ne veut reconnaître ni la gravité, ni même la réalité ? Certes, à titre purement 
individuel, le pardon "gratuit», unilatéral, est toujours possible. Mais un tel pardon " christique » échappe complètement au registre politique : il engage l'individu dans son for intérieur, le dépouille de sa capacité à faire voix. À l'échelle de nos sociétés, le pardon ou la demande de pardon ne sauraient faire sens s'ils passaient outre une juste appréciation de la réalité et de la gravité des faits, s'ils ne s'accompagnaient d'un vaste mouvement de remembrance qui heurte l'indolence ou l'intangibilité du souvenir. En somme, la reconnaissance est le versant sociétal du pardon moral, sa cristallisation politique et pragmatique, son inscription temporelle. Hors cette solidarité pratique avec la notion de reconnaissance, la notion de pardon serait d'essence, d'inspiration essentiellement morale, relevant d'un au-delà de la justice des hommes, de l'ordre de la cité céleste plutôt que de l'ordre des choses ici-bas. C'est pourquoi, au niveau sociétal, l'acte et la symbolique de la reconnaissance sont la condition sine qua non de l'acte et de la symbolique du pardon; autrement dit, l'acte de pardon, s'il n'est lui-même annoncé - comme « ourdi », tramé - par l'acte et la symbolique de la reconnaissance, se trouve précisément dépourvu de tout ressort symbolique autre qu'étroitement moral, donc de toute efficacité politique. Dès lors, toute politique de la main tendue, si elle aspire sincèrement à transcender la portée du geste, à revêtir sa mécanique de signification ${ }^{35}$, ne doit pas viser à clore un chapitre mais à l'ouvrir, et à l'ouvrir ensemble. En l'espèce, il s'agirait de restituer la trame, la toile de fond sur laquelle se détache le drame de la disparition des harkis, d'opérer la mise en intrigue des faits, de leur offrir une résonance ; il s'agirait, en somme, de rétablir - à l'échelle des sociétés algérienne et française - l'assise matérielle et l'épaisseur symbolique des faits. Le contre-exemple institutionnel de la loi de " concorde civile » À cet égard, bien que les responsabilités ayant trait au déclenchement et au pourrissement de la seconde guerre d'Algérie soient moins aisément discernables que celles ayant trait au massacre des harkis ${ }^{36}$, la loi de « concorde civile » promulguée le 16 juillet 1999 en vue de « dégager des issues appropriées aux personnes impliquées et ayant été impliquées dans des actions de terrorisme ou de subversion $\|^{37}$ n'est certes pas en mesure de créer un précédent institutionnel. Tant la lettre que l'esprit de la démarche générale de la présidence algérienne ressortissent non d'un acte de pardon ou d'un processus de réconciliation au sens politique du terme ${ }^{38}$ mais d'un processus unilatéral de réconciliation au sens religieux du terme ${ }^{39}$; non un processus présidant au rétablissement de la concorde civile, laquelle suppose l'aménagement d'un espace de réciprocité où puisse être énoncé - et non effacé - le différend, mais le «fait du prince ", une grâce amnistiante conditionnée par un acte de capitulation en bonne et due forme ${ }^{40}$, un "octroi» en forme d'ultimatum ${ }^{41}$. Par conséquent, il ne s'agit pas de négocier avec l'adversaire, mais, après s'être assuré de son " amendement entier $»^{42}$, de faire acte de mansuétude à son égard ; autrement dit, de l'amener à résipiscence contre la promesse d'une impunité relative (et la certitude d'une impunité totale pour soi). De fait comme de droit, la loi de "concorde civile» ressortit moins de l'ordre de la délibération que de celui de l'expédient, au double sens d'user d'expédients ${ }^{43}$ et de vivre d'expédients ${ }^{44}$. Ce primat de l'amodiation sur la négociation appert de l'article 1er de la loi dite de «concorde civile», lequel dispose qu'il s'agit de «dégager des issues appropriées " non à la situation mais "aux personnes impliquées ou ayant été impliquées dans des actions de terrorisme ou de subversion ». De toute évidence, cette loi s'attache moins à réunir les conditions d'un règlement global du conflit qu'à réunir celles, dissolvantes, d'un sauf-conduit généralisé ${ }^{45}$. Elle ne saurait dès lors satisfaire ni les victimes civiles du terrorisme islamiste ${ }^{46}$, ni les familles de disparus. Pas plus qu'elle 
ne saurait satisfaire l'aspiration de l'ex-F.I.S. (dissous) à être réintégré dans le jeu politique. Ainsi, pour Cherifa Kheddar, qui a fondé une association de victimes suite à l'assassinat de plusieurs membres de sa famille par des islamistes armés, « [la loi sur la " concorde civile »] blanchit les terroristes sans véritable procès. On devrait même assigner l'État qui ne nous a pas protégés. Il faudrait en tout cas que nous puissions avoir un débat public sur ce qui s'est passé, et pas simplement tourner la page $»^{47}$. Pour antagoniques que fussent leurs univers de justification et leurs ordres d'engagement, c'est le même type de réserves et de récriminations qui ressortaient des propos d'Abdelkader Hachani, $n^{\circ} 3$ du F.I.S., peu avant son assassinat le 22 novembre $1999^{48}$. Pointant le caractère «ambigu » de la démarche référendaire d'Abdelaziz Bouteflika, tant du point de vue du libellé de la question ${ }^{49}$ que de celui de la limitation dans le temps des bénéfices attachés à la loi de "concorde civile", Abdelkader Hachani craignait que «cette manière de procéder serve à lui donner un alibi pour le déclenchement d'une action répressive plus forte encore que celle qui a eu lieu jusqu'à présent ». Il appelait à ce que "soient jugés, dans le camp du pouvoir comme dans l'autre, ceux qui ont commis des crimes. Nous ne pourrons nous réconcilier que si les responsabilités de chacun sont clairement établies. Quand je saurai qui a tué mon frère, je pourrai lui pardonner »; " Mais, une fois encore, ajoutait-il, j'ai le regret de croire que la violence va continuer, car on ne s'attaque pas aujourd'hui à ses causes $\aleph^{50}$. De fait, le pardon, loin d'être une formule illocutoire «qui dit ce qu'elle fait et qui fait ce qu'elle dit ", loin d'être une fin en soi et une fin tout court, un trait tiré sur le passé circonvenant à toute forme d'examen de conscience, est bien au contraire une prise de risque assumée et partagée. Il s'agit, par-delà le ou les discours de l'identification sécurisante, de mettre à nu, de déconstruire et d'offrir à la critique - la sienne propre et celle de son vis-à-vis - la résonance propre à la mémoire du différend. Il s'agit, surtout, d'en démystifier les totems et les tabous, ce surcroît de crispation sur les identités que sont la fixation, la surenchère et / ou l'amnésie. Ainsi, loin d'être " une petite affaire de morale ou de religion personnelle ${ }^{51}$, une échappatoire, un moyen terme, le pardon est et se doit d'être, outre une anamnèse véritable, une remise en cause de cet excès d'identité que fonde le tragique de conflit. Aussi bien le pardon participe-t-il, là où l'histoire touche au tragique, de la nécessaire mise en cause des rapports sociaux qui ont fondé l'exclusion et la stigmatisation de la victime, et de celle, non moins nécessaire, des valeurs et vecteurs symboliques qui légitiment ces rapports sociaux aux yeux de l'offenseur. « [Le pardon], écrit Olivier Abel, libère d'une excessive obsession de l'identité. Avec lui la mémoire n'est plus l'interminable récit du passé, ou plus exactement l'interminable garantie d'une identité $»^{52}$. Il introduit une altération dans l'identité même, «il touche à l'identité en tant qu'elle est ancrée dans un souvenir qu'elle répète, ou dans un oubli désormais sacré [i.e. institutionnalisé] $»^{53}:$ c'est respectivement assez bien le cas pour l'identité de la communauté harkie par rapport au martyre enduré au crépuscule de la guerre d'Algérie, et pour l'identité de la République algérienne fondée sur la table rase de ce qui la précédait. Au fond, le pardon, en ce qu'il peut - en ce qu'il doit - être envisagé comme un "acte éthique d'autonomie », est une forme d'acceptation. Au regard de l'agresseur, c'est la part de la reconnaissance. Il s'agit pour lui d'accepter de faire retour au passé, au passé tel qu'il pèse, c'est-à-dire de faire retour à cette part de l'événement qui heurte la geste de l'identification sécurisante; en somme, il s'agit de réinvestir, en même temps que le champ du réel, le champ de la responsabilité et d'être en mesure de répondre de ses actes. Au regard de la victime, il s'agit d'accepter la réalité de la perte, d'accepter le 
tragique de l'irréversible. «En désignant l'irréparable, l'intraitable, ce qu'on ne peut pas raconter entièrement, écrit Olivier Abel, le pardon accepte qu'il y a de la perte. Il fait ce travail de deuil sans lequel il n'y a pas de travail d'enfantement ou de résurrection possible d'un autre présent $\aleph^{54}$. Condition même de la sublimation, le pardon scelle chez la victime l'acceptation, le réinvestissement d'un présent dont le ressentiment et le désir de vengeance l'avaient tenue éloignée. Algérie : de la difficulté de s'inscrire dans une démarche de pardon sans altérer les frontières de l'identité Accepter de demander pardon aux harkis pour les massacres de l'été et de l'automne 1962, ce n'est pas, pour les autorités algériennes, déconsidérer le sens de l'engagement du F.L.N. en faveur de l'accession à l'indépendance de l'Algérie; c'est accepter de reconsidérer, au sens le plus pragmatique du terme, l'histogenèse de cet engagement, de déparer l'engagement de son légendaire pour s'attacher aux faits. Au fond, il s'agit d'en accepter la relativité, la part de contingence, d'arbitraire même. En l'espèce, accepter de demander pardon aux harkis pour les massacres de l'été et de l'automne 1962, ce n'est pas, pour les autorités algériennes, se déjuger, ni «faire amende honorable " (et d'ailleurs qu'est-ce à dire là où le mal est irréparable, de toutes les façons inexpiable ?55). C'est reconnaître, en revanche, que l'engagement armé du F.L.N. en faveur de l'indépendance de l'Algérie a suscité, au sein même de cette part de la population dont le F.L.N. revendiquait l'exclusivité de la représentation, une adversité qui avait sa raison d'être ${ }^{56}$, méconnue en son temps puisque combattue non par des voies démocratiques mais au prix d'une violence sans langage ${ }^{57}$. Ce serait, en somme, reconnaître que l'adversité ne devrait exclure ni la lucidité ni la réciprocité, autrement dit, récuser cette propension récurrente des vainqueurs à ajouter l'hégémonie à la victoire, à écrire l' «Histoire » de leur seul point de vue et à leur seul profit moral. Ce serait par là reconnaître que l'advenu n'est, en dernière analyse, que l'issue contingente d'un rapport de forces, et qu'il appartient aux hommes - à tous les hommes - et non à l'« Histoire $»^{58} \mathrm{~d}$ 'y voir ou non la réalisation de l'idée de justice. De ce point de vue, force est de constater que les conditions du pardon, c'est-à-dire de l'acceptation, ne sont pas réunies. La trame de la disparition des harkis s'articule invariablement sur le mode de l'obstruction et de la dissonance plutôt que sur le mode de la reviviscence et de la reconnaissance. En Algérie, l'occultation des faits participe d'une vision obsolescente et dogmatique du monde, fortement empreinte d'historicisme et de messianisme. Elle ressortit à la fois d'un rapport onirique, fantasmatique au passé et d'une idéologie de l'enfermement qui en est comme le corollaire. Ainsi, le récit de la guerre de libération nationale et le mythe selon lequel l'indépendance fut conquise au terme d'une victoire militaire de l'A.L.N. ${ }^{59}$ sert, aujourd'hui même en Algérie, à justifier la mainmise de l'A.N.P. ${ }^{60}$ sur l'appareil d'État. De la même manière, le mythe de l'unanimité des masses derrière le F.L.N. durant la guerre d'Algérie commande à la fois d'occulter, dans son historicité propre, la destinée tragique des harkis ${ }^{61}$, et d'instrumentaliser, en la réifiant, la figure du harki, figure éponyme du traître, bouc émissaire évident pour tous les maux qui touchent l'Algérie indépendante et, à ce titre, échappatoire commode pour la caste militaire au pouvoir ; figure-repoussoir aussi, qui sert de défouloir « ex-post » aux masses algériennes, vidant l'abcès illégitime, refoulé, de l'indécision durant la guerre d'Algérie ${ }^{62}$. S'agirait-il d'« éradiquer » les groupes islamistes armés, leurs membres seraient assimilés à « des enfants de harkis [qui] viennent de France pour tuer des moudjahidin au nom du G.I.A. et [qui] cherchent à venger leurs traitres de pères ${ }^{63}$. S'agirait-il, au contraire, de se concilier les islamistes armés, on jugerait des conditions tant de leur possible 
rédemption que de leur possible acceptation ${ }^{64}$ à l'aune de ce "contre-étalon " symbolique (dans l'imaginaire social) et de ce contre-exemple pratique (pour les autorités) que sont respectivement l'engagement des harkis et le sort qui leur fut réservé à l'indépendance. Pour Mohamed, un ancien moudjahid qui s'est porté à la tête du groupe des patriotes de Chefka (420 km à l'est d'Alger) ${ }^{65}$, "[les combattants des groupes islamistes armés] ne sont pas comme les harkis, qui avaient pris le parti des Français. Les groupes armés ne se battent pas contre le pays, mais contre certains hommes. Ce sera plus facile de les accepter à nouveau $»^{66}$. Sans pour autant qu'il soit possible d'y dénoter l'expression d'une adhésion sincère et réfléchie à la politique de " concorde civile ", le discours des hommes de base du système fait remarquablement écho à celui des hiérarques. Dans une interview accordée à France Culture le 12 septembre 1999, Abdelaziz Bouteflika assurait ainsi que « la responsabilité [des crimes], en aucune manière, ne saurait être collective ${ }^{67}$. Quant à l'esprit de fronde qui semble animer les officiers dissidents du M.A.O.L. ${ }^{68}$, il semble peu à même d'inaugurer une ère de plus grande transparence qui verrait la redéfinition des rapports entre l'institution militaire et les instances politiques. En effet, pour virulents que soient les termes de la polémique, ils participent d'une homologie structurale flagrante (et non d'une rupture épistémologique) avec les canons de la propagande d'État. Ainsi, quoiqu'il préconise « la convocation d'une conférence nationale pour la vérité et la réconciliation [sur le modèle sud-africain ?] » et qu'il considère que «la crise ne peut trouver une solution par des "semblants" d'accords mais par la recherche de compromis $"{ }^{69}$, le M.A.O.L. n'use, pour fustiger les caciques de l'A.N.P., d'autre registre polémique que celui, éculé et démagogique, de la thématique " harkie " : les principaux chefs de l'A.N.P. seraient des «nouveaux harkis» dont l'avènement "reconduit [l'Algérie] à l'heure de l'occupation $»^{70}$, des "généraux harkis » et / ou des «traîtres harkis » qui ne représentent que « le retour rampant au colonisateur d'hier $»^{71}$, ou encore « des harkis algériens [qui] ont utilisé la solution finale (sic) contre de braves Algériens, hommes et femmes $»^{72}$. Le récit hypostasié de la guerre de libération nationale, fruit d'un « cadrage intense ", principiellement exclusif ${ }^{73}$ et idéellement indivis ${ }^{74}$, n'en finit pas de servir de "loi narcissique » à cette Algérie que l'on disait autrefois «nouvelle» ou "postcoloniale ", mais qui, de fait, n'a jamais cessé de vivre dans « une sorte de panique identitaire qui s'exprime dans [une] violence "élémentaire" - [cette violence élémentaire] qui questionne les éléments constituants d'un collectif, et le ramène à cette scène "primitive" où l'on sacrifie de l'humain à la déesse primordiale $»^{75}$. Si " certains traits, devenus symptômes, sont propres à l'espace algérien et à son impasse [symbolique] actuelle ", nul doute que le caractère obsessionnel de l'instrumentation à des fins polémiques d'irrévérences telles que "parti de la France» ou "nouveaux harkis » ne trahisse le rôle outré imparti à la manipulation de la mémoire, à la fois « instrument et objectif de puissance ${ }^{76}$. Ainsi, en Algérie, la représentation de la figure du harki navigue entre les écueils normativement contradictoires de l'évocation et de la symbolisation. À la fois "figure-totem ", emblématique de cette forme insidieuse de l'altérité qu'est la "traîtrise », et figure taboue, dont il s'agit de nier l'historicité dans une Algérie nouvelle que l'on eût voulue pareille à une table rase, la figure du harki dessine une geste foncièrement ambivalente : elle est susceptible tant de servir que de desservir les desseins de l'imagerie officielle selon qu'elle soit dépeinte selon les traits nécessairement complexes et circonstanciés de sa destinée ou revisitée à l'aune des pré-requis de la vulgate révolutionnaire et / ou nationaliste. D'où l'entre-deux paradoxal d'une propagande d'État sommée de faire en sorte que la figure du harki ne 
soit pas considérée comme étant "des nôtres ${ }^{77}$ tout en accréditant l'idée qu'elle "nous » menace spécifiquement. Dans cette optique, tout l'effort de la propagande d'État doit tendre à rendre la figure du harki à la fois méconnaissable (sociologiquement altère) et reconnaissable (idéologiquement familière). La figure incarnée du harki est ignorée pour être hypostasiée en une représentation socialement générique de l'ennemi intérieur. Les trajectoires vécues des supplétifs de l'armée française sont bannies hors du cadre d'appréhension et de compréhension de l'Algérie nouvelle, pour être uniment réduites à quelque résidu, à quelque aberration redevables du glacis colonial, parenthèse dont on s'interdit par principe d'apprécier la résonance (pour ne pas dire les apports). La figure du harki ne doit en aucune façon être une figure d'évocation dont la trame servirait l'analyse de la complexité algérienne : elle n'a d'usage qu'instrumental, que totémique. Elle est La figure d'excommunication par excellence. France : de la difficulté de faire sourdre un passé occulté sans interroger les fondements de la raison d'État En France, l'idée d'une reconnaissance officielle de la responsabilité de l'État français dans l'abandon des harkis ${ }^{78}$ se heurte à l'intangibilité d'une imagerie d'État rétive à toute forme d'examen de conscience relativement aux grands conflits de ce siècle. Certes, cette revendication se fonde sur un précédent : la reconnaissance par Jacques Chirac de la responsabilité propre de l'appareil d'État français dans la politique de persécution et dans la déportation des Juifs sur et à partir du territoire national entre 1940 et 1944 . Toutefois, pour au moins deux raisons, elle n'est sans doute pas susceptible d'être satisfaite à court ou moyen terme. D'une part, elle mettrait en jeu la réputation, désormais consensuelle, du général de Gaulle. D'autre part, une telle entreprise participerait nécessairement de la remise en cause des fondements pragmatistes de la raison d'État, dont l'illustration archétypique est précisément la conclusion des accords d'Evian, à la fois témoignage et condition de la "grandeur » retrouvée si l'on en croit l'historiographie officielle. À cet égard, le fait que ne soit stipulée dans les textes la moindre menace de sanction effective en cas de violation de la Déclaration des garanties contenue dans les accords d'Evian est symptomatique de la contingence des considérations humanistes dans le discours de la méthode gaullienne. Car, ces dispositions générales ${ }^{79}$ étaient les seules à même de garantir la sécurité et la liberté de circulation des Musulmans pro-français en l'absence de chapitre spécifique consacré au devenir de cette population. De fait, relève Guy Pervillé, « les garanties de la sécurité des personnes furent l'objet des violations les plus précoces et les plus graves. La lutte contre l'O.A.S. et l'anarchie de l'été 1962 déclenchèrent une vague d'enlèvements de Français suivis d'assassinats ou de séquestrations, en dépit de la présence des troupes françaises (...). En même temps, des milliers d'anciens supplétifs musulmans ("harkis" et autres catégories) de l'armée française, ainsi que d'anciens élus, furent victimes de cruelles représailles, tôt ou tard suivant les régions. Les efforts de l'Association des anciens officiers des Affaires algériennes pour les transférer en métropole en 1962 se heurtèrent à l'obstruction du gouvernement français. Des bilans différents estiment le nombre des victimes à 30000 ou à 150000 par extrapolation à partir de témoignages localisés ${ }^{80}$. "Pour le pragmatisme, est vrai ce qui réussit », peut-on lire dans le Petit Larousse. Le critère de la vérité qui, du côté français, a présidé à la conclusion « en l'état » des accords d'Evian se mesure donc, dans l'esprit des négociateurs français, à l'aune de la valeur pratique de la politique de "dégagement » visée et entreprise. Une telle politique commandait de découpler les dispositions touchant aux intérêts stratégiques de la France de celles relatives à la sécurité des personnes, de loin les plus sensibles et les plus lourdes à 
mettre en œuvre. Car celles-là seules, semble-t-il, engageaient la " grandeur » nationale dans l'esprit de nos gouvernants. La politique de "dégagement» impliquait, par conséquent, que la France se désengage de l'application des dispositions relatives à la sécurité des personnes ${ }^{81}$ et s'en remette, en l'espèce, au bon vouloir du futur État algérien. Aussi, pour solennelles et rassurantes que furent les exégèses officielles au moment de la conclusion des accords d'Evian, jamais l'armée française - qui stationna pourtant en Algérie jusqu'en juin 1964 - ne reçut l'ordre d'intervenir pour secourir ceux qu'elle avait irrémédiablement « compromis ». Pas davantage, elle ne reçut l'ordre d'intervenir pour secourir les milliers de pieds-noirs enlevés après l'indépendance et qui, à l'instar des harkis, connurent un sort effroyable. «Les intérêts stratégiques de la France, écrit Guy Pervillé, furent de loin les mieux respectés (...). Les dernières troupes de l'armée de terre furent évacuées en juin 1964 et toutes les bases concédées [par l'Algérie] furent rendues avant terme, au moment où le gouvernement français avait cessé de les juger indispensables (les sites sahariens en 1967, Mers El-Kebir en 1968, Bou Sfer en 1970) ». Cependant, ajoute-t-il, "l'honneur [de la France] a, dans le même temps, souffert de l'abandon de trop de ses ressortissants et de ses partisans à des vengeances prévisibles. Les intérêts des Français d'Algérie ont été sacrifiés à ceux (énergétiques et stratégiques) de la métropole $»^{82}$. Entendons-nous bien : la geste officielle de la guerre d'Algérie ne peut à la fois reprendre à son compte le discours de la méthode gaullienne, fondée sur un « pragmatisme » qui confine au machiavélisme, et rendre compte de la trame de la disparition des harkis qui, précisément, implique de faire retour sur la mise entre parenthèses des valeurs morales qui a présidé à la politique de "dégagement». Plutôt que d'assumer cette contradiction, l'historiographie officielle s'emploie depuis lors à occulter le tribut effroyable payé par les harkis à la réorientation de la politique de grandeur de la France. Autrement dit, l'explication globalisante selon laquelle le général de Gaulle aurait réinscrit la France dans le sens de l'Histoire et préservé ses intérêts vitaux ${ }^{83}$ en parachevant le processus de décolonisation en général, en dénouant le drame algérien en particulier, n'est recevable que si elle s'abstrait des conséquences humaines, somme toute exorbitantes, imputables aux modalités pratiques d'exercice de cette politique. Par-delà même les hypothèques attenantes à la raison d'État, et pour rendre la pleine mesure des logiques symboliques de la stigmatisation des anciens supplétifs de l'armée française, il faut également évoquer l'incongruité de la figure du harki dans la geste des intellectuels français en guerre d'Algérie. En soi, la destinée des harkis, depuis l'engagement jusqu'à la mort ou l'exil, est un élément pivot de la complexité algérienne qui aurait dû susciter sinon la sollicitude, du moins l'intérêt des intellectuels en guerre d'Algérie. Si tel n'a pas été le cas, c'est qu'au travers du prisme de la littérature tiers-mondiste, la figure du harki est une figure malvenue, idéologiquement alogique et politiquement illogique, sinon illégitime, un corps étranger au "mouvement de l'Histoire ". Le pont aux ânes des prêcheurs d'abstraction, en somme ${ }^{84}$. Si nous devions, en l'espèce, qualifier les rapports que la France entretient avec son passé et avec ceux qui en furent les principales victimes, nous en appellerions à la pusillanimité et au pharisaïsme. Comment, dans ces conditions, la communauté harkie pourrait-elle pardonner, et à qui, puisque ni l'Algérie ni la France ne veulent reconnaître officiellement la réalité et la gravité des faits, puisque ni l'Algérie ni la France ne semblent prêtes à assumer officiellement leur part de responsabilité dans la destinée tragique des harkis, puisque ni l'Algérie ni la France ne semblent devoir considérer venu le temps de la remise à plat, et, pour tout dire, de la remise en cause de leurs gestes officielles respectives 
relativement à la mémoire de la guerre d'Algérie? Pourquoi la communauté harkie devrait-elle pardonner, et à qui, puisque ni l'Algérie ni la France ne lui demandent pardon?

\section{NOTES}

1. Paul Thibaud, «Algérie : faut-il prolonger la guerre des mémoires? », Esprit, n²71, janvier 2001, p. 198. C'est l'auteur qui souligne.

2. Ibid.

3. Ibid, p. 199.

4. Cf. Emmanuel Brillet, Mémoire, identité et dynamique des générations au sein de la communauté harkie. Une analyse des logiques sociales de la stigmatisation, thèse de doctorat en cours d'élaboration (sous la direction de John Crowley) au sein du Centre de recherches et d'études politiques (C.R.E.D.E.P.) de l'université Paris IX-Dauphine.

5. S'agissant de la destinée des harkis et de leurs familles depuis l'engagement jusqu'à la mort ou l'exil, cf. Emmanuel Brillet, « La contingence et la geste : le harki, l'indicible du "mouvement de l'histoire" ", in Alain Brossat et Jean-Louis Déotte (dir.), L'époque de la disparition. Politique et esthétique, Paris, L'Harmattan, 2000.

6. Pour une analyse plus resserrée des us et abus de la thématique harkie par les protagonistes de la seconde guerre d'Algérie, cf. Guy Pervillé, « Histoire de l'Algérie et mythes politiques algériens : du "parti de la France" aux "anciens et nouveaux harkis" ", in Charles-Robert Ageron (dir.), La guerre d'Algérie et les Algériens 1954-1962, Paris, Armand Colin / Institut d'histoire du temps présent (CNRS), 1997, pp. 323-332.

7. Dans son dernier ouvrage, Paul Ricœur fait de l'expression publique du dissensus, guidée par l'esprit de pardon, la condition de « la salutaire crise d'identité permettant [au niveau de la mémoire privée et de la mémoire collective] une réappropriation lucide du passé et de sa charge traumatique »; cf. Paul Ricœur, La mémoire, l'histoire, l'oubli, Paris, Editions du Seuil, 2000, p. 589.

8. En France et en Algérie.

9. Daniel Sibony, « Algérie : une étrange violence », Libération, édition du 6 avril 1998.

10. Ibid.

11. Ibid.

12. Ainsi, au cours de la campagne électorale précédant les élections présidentielles du 16 novembre 1995, le général-candidat Liamine Zéroual, faisant référence aux violences qui meurtrissent actuellement l'Algérie, avait déclaré (sans étayer le moins du monde ses allégations) que « la plupart des criminels et des mercenaires sont des harkis ou des fils de harkis, soutenus et financés par des puissances étrangères et qui ont choisi la destruction de leur pays » (cité in El Watan du 15 novembre 1995).

13. Paul Thibaud, «Génération algérienne? », Esprit, n¹61, mai 1990, p. 49.

14. Ibid.

15. Entendre O.A.S. et « soutiens».

16. On pourrait en dire autant de la mémoire de l'abandon, puis de l'exil. 
17. Olivier Abel, «Ce que le pardon vient faire dans l'histoire », Esprit, $\mathrm{n}^{\circ} 193$, juillet 1993 , p. 62.

18. Ibid, p. 63.

19. Ibid.

20. Fédération Nationale des Anciens Combattants d'Afrique du Nord en Algérie, au Maroc et en Tunisie, originellement d'obédience communiste.

21. Assassinats et enlèvements respectivement consécutifs de la guerre à outrance menée par l'O.A.S. contre les troupes régulières de l'armée française (et réciproquement) jusqu'à la mi-juin 1962 ; des débordements incontrôlés (à plus d'un titre, et notamment en raison de la passivité délibérée de l'armée française) du 5 juillet 1962 à Oran (où plusieurs centaines de pieds-noirs furent massacrés) ; consécutifs, enfin, des Saint-Barthélemy incessantes orchestrées par le F.L.N. à l'encontre des musulmans pro-français jusqu'au début de l'année 1963.

22. Olivier Abel, op. cit. p. 63.

23. Ibid, pp. 64-66.

24. Ibid, pp. 60-61.

25. Ibid, p. 64.

26. Ibid, p. 61.

27. Ibid, p. 72.

28. Ibid, p. 62.

29. Olivier Abel (dir.), Le pardon, briser la dette et l'oubli, Paris, Autrement, 1991. Cet ouvrage propose précisément une mise en perspective intéressante des différents apports des sciences sociales sur la question du pardon, et s'attelle notamment à l'étude de cas paradigmatique du différend opposant Turcs et Arméniens sur la question de la reconnaissance des massacres de 1915.

30. De fait, à l'échelle d'un massacre collectif, nulle réparation ou compensation n'est susceptible de « purifier » (du latin expiare) le crime commis, c'est-à-dire littéralement de le débarrasser de la souillure morale dont il est entaché. Dès lors, à la différence de l'expiation, le pardon ne saurait s'appliquer aux faits (car leurs conséquences sont irréversibles, non susceptibles d'être réparées) mais, plus modestement (et plus pragmatiquement), à la relation des faits, c'est-à-dire à la manière de considérer collectivement les faits et de considérer collectivement l'autre - la victime ou l'agresseur - considérant les faits. De la part de l'agresseur, accepter de demander publiquement pardon à la victime, c'est s'astreindre à assumer le crime. Prétendre l'expier, c'est entretenir l'illusion de pouvoir l'effacer. L'emploi du qualificatif « inexpiable » vise donc ici tant à objectiver la logique sociétale à l'œuvre dans l'ordonnancement du massacre des harkis qu'à contester à l'agresseur le droit de se défausser de ses responsabilités au nom d'une conception eschatologique du pardon. L'acception religieuse de la notion d'expiation, contiguë de l'idée qu' « ici-bas tout s'expie », part en effet du principe que la vie terrestre n'est qu'une étape et que, conséquemment, il n'y a de faute ou de crime dont les conséquences ne soient réversibles. Elle privilégie, dès lors, l'acte de pénitence - qui vise à réparer l'offense faite à Dieu - sur l'acte de reconnaissance de la réalité et de la gravité des faits : dans l'idée d'expiation, c'est le Jugement dernier qui importe, non le jugement des contemporains

31. Olivier Abel, op. cit., p. 72.

32. Ibid, p. 70. 
33. Au double sens du terme " effet(s) ", soit, d'une part, l'influence des faits sur l'être de la victime, et, d'autre part, les procédés d'influence sur les faits dont use l'agresseur. 34. Daniel Sibony, op. cit.

35. Autrement dit, à en faire non un geste commode, un «pardon » par le geste (une politique de la page blanche : " on se serre la main et on oublie tout»), mais une geste incommode, une geste de pardon qui soit non une « chanson de geste(s) » mais un récit bipartite qui s'attache à écailler tout vernis épique, et où la poignée de main (symbolique) procède de (et non précède) l'échange de paroles, où l'échange de paroles - et lui seul - fait symbole

36. Le massacre des harkis est imputable sans équivoque possible à l'arbitraire d'éléments placés sous l'autorité de fait ou de droit du F.L.N.. Il a été perpétré unilatéralement et illégalement (c'est-à-dire en violation manifeste de la clause de nonreprésailles contenue dans les accords d'Évian) à l'encontre d'éléments démobilisés et rendus désarmés à la vie civile (donc censément placés sous la protection de ceux-là mêmes qui se sont rendus responsables de leur supplice). A l'inverse, les islamistes armés sont placés en état de rébellion ouverte. En outre, chacun des protagonistes de la crise ouverte en 1992 peut avoir intérêt - dans l'optique d'affirmer ou de voir réaffirmée son hégémonie (car tel est bien l'enjeu de la lutte en l'absence de jeu démocratique) - à pratiquer la politique du pire

37. Article 1er de la loi de " concorde civile », chapitre 1 : «Dispositions générales ». 38. C'est-à-dire au sens où l'on en vient à « [construire] un mixte entre plusieurs langages, plusieurs univers de justification (...) sans trancher définitivement sur le fond » (Olivier Abel, op. cit., p. 65).

39. Soit une « cérémonie par laquelle un pécheur est pardonné et réadmis à la communauté par l'église » (Petit Larousse, 1993, p. 863).

40. Pour bénéficier des dispositions gracieuses contenues dans la loi de " concorde civile », les personnes visées doivent « aviser les autorités compétentes qu'elles cessent toute activité de terrorisme et se présenter à ces autorités » (art. 1er, chapitre 1 : «Dispositions générales ») ; elles sont également tenues de "faire une déclaration qu'elles attestent sincères de l'armement, explosifs, munitions et moyens matériels en leur possession et de les remettre aux autorités auxquelles elles se seront présentées, la même déclaration [devant] comporter, en outre, les actes qu'elles ont commis ou auxquels elles ont participé » (art. 8, chapitre 3 : « De la mise sous probation »); bien plus, seront éligibles au régime de la mise sous probation « les personnes qui se seront admises à participer, sous l'autorité de l'État, à la lutte contre le terrorisme » (art. 8, chapitre $3:$ : De la mise sous probation »).

41. Les personnes visées ne peuvent bénéficier des mesures contenues dans la loi que " dans un délai de six mois à compter de [sa] promulgation [celle-ci étant intervenue le 13 juillet 1999] » (art. 3, chapitre $2:$ « De l'exonération des poursuites »).

42. Art. 6 de la loi de " concorde civile», chapitre $3:$ « De la mise sous probation ». 43. L'expédient étant défini, en ce sens, comme un «moyen de résoudre momentanément une difficulté, de se tirer d'embarras » (Petit Larousse, 1993, p. 419).

44. Au sens de « recourir à toutes sortes de moyens, licites ou non, pour subsister » (Ibid).

45. Il s'agit moins, en somme, de faire la paix que d'avoir la paix. En cela, nous souscrivons en plein à l'analyse du journaliste Djallal Malti qui, prenant acte du fait qu'Abdelaziz Bouteflika avait exclu « d'ouvrir des dossiers [ou de] faire des chasses aux sorcières [relativement aux agissements de l'armée depuis le début de la crise] », 
considère que le référendum sur la « concorde civile » « représente le meilleur moyen, aux yeux des militaires, de jeter un voile pudique sur la politique "sécuritaire" engagée en 1992 »; « Pour les militaires, ajoute-t-il, il s'agit en premier lieu de s'assurer de l'impunité pour tous les crimes et les violations des droits de l'Homme commis depuis lors » (Djallal Malti, « Les deux faces de la concorde algérienne », La Chronique d'Amnesty, novembre 1999, pp. 14-15)

46. Lequel, par surcroît, est quelquefois un terrorisme d'État déguisé.

47. Témoignage cité in Florence Aubenas, « On change de rêve, pas de réalité », Libération, mercredi 15 septembre 1999, p. 10.

48. Le colonel Baali Ali, l'un des porte-parole du M.A.O.L. (Mouvement algérien des officiers libres, voir infra), impute l'assassinat d'Abdelkader Hachani aux plus hauts responsables de l'état-major de l'A.N.P. : « En éliminant cet ultime "politique" du F.I.S., interlocuteur incontournable et dernière passerelle vers un règlement de fond de la crise, les jusqu'au-boutistes du pouvoir écartent toute perspective d'un règlement politique du conflit en consacrant des accords sécuritaires secrets »(Y.B. [anonyme] et Samy Mouhoubi, «Algérie : un colonel dissident accuse », Le Monde, samedi 27 novembre 1999, pp. 14-15).

49. La question à laquelle étaient appelés à répondre les électeurs algériens était : «Etes-vous pour ou contre la démarche générale du président de la République visant à la réalisation de la paix et de la concorde civile ? ». Ce libellé met en balance non pas tant une manière de faire la paix (c'est-à-dire une manière de faire la paix parmi d'autres) que l'idée même de faire la paix, comme si celle-ci était instamment attachée à - ou était redevable de - l'avènement d'Abdelaziz Bouteflika à la présidence de la République algérienne. Florence Aubenas, qui rappelle que «seuls les partisans du oui ont été autorisés à faire campagne », note avec justesse que, « compte tenu de l'aspiration des Algériens à la paix et du libellé de la question », « ce référendum ressemble plus à un plébiscite qu'à une consultation démocratique » (« Un plébiscite pour le Président », Libération, jeudi 16 septembre 1999, p. 9).

50. Propos recueillis par Florence Aubenas, art. cit.

51. Olivier Abel, op. cit., p. 72.

52. Ibid, p. 71.

53. Ibid, p. 70.

54. Ibid.

55. Entendons-nous bien, à nouveau, quant à ce que nous considérons être ou non du domaine de l'inexpiable. Certes, le fait du massacre des harkis n'est pas susceptible d'être réparé : il demeure et demeurera toujours un des événements fondateurs de l'Algérie nouvelle, son " péché originel » en quelque sorte. Cependant, l'état du rapport des forces (sociales, politiques et institutionnelles) qui y a présidé, ainsi que le type de justifications dont il est entouré, sont eux réformables. C'est là la part dévolue à la demande de pardon : non une réparation des faits (l'agresseur, s'il peut se défaire de sa morgue, ne peut ressusciter les morts), mais une réparation des termes de l'échange. L'hégémonie politique de la camarilla militaire en place, qui perpétue sans discontinuer depuis 1962 les pratiques autoritaires et violentes qui conférèrent au F.L.N. / A.L.N. son leadership dans le combat pour l'indépendance, n'est pas une fatalité. Mais elle ne pourra être contestée avec succès qu'à la condition expresse que soient revisités les mythes fondateurs qui servent de caution symbolique aux « Grands Anciens ».

56. Ce qui revient, pour la partie algérienne, à relativiser et à (re)contextualiser son engagement propre, à récuser l'emprise de la geste sur les faits, à départir la geste 
officielle de ses accents messianiques et à reconnaître la contingence du rapport de forces, son indétermination morale, autrement dit, à rendre sa part d'historicité à l'adversaire.

57. Il convient de rappeler, à cet égard, que la guerre d'Algérie, loin d'être uniquement (et uniment) une guerre de décolonisation, fut (déjà) une guerre civile puisque, entre le 1er novembre 1954 et le 19 mars 1962, le ratio des victimes civiles du F.L.N. (ventilées selon leur communauté de rattachement) est en étroite corrélation avec le rapport démographique qui prévalait alors en Algérie entre les communautés musulmane et européenne : il y eut ainsi 3663 victimes civiles européennes des actes de terrorisme du F.L.N. (2788 tués, 875 disparus) pour 29674 Musulmans (16378 tués, 13296 disparus), soit un Européen pour huit Musulmans. Ce ratio, établi pour la période comprise entre le déclenchement de l'insurrection et la proclamation du cessez-le-feu en Algérie, ne tient pas compte des dizaines de milliers d'anciens harkis massacrés par le F.L.N. après la proclamation du cessez-le-feu et l'accession à l'indépendance de l'Algérie. Il ne tient pas davantage compte des 3957 Musulmans victimes du terrorisme du F.L.N. et des règlements de compte internes à la mouvance nationaliste en métropole (bilan partiel établi pour la période courant du 1-1-1956 au 23-1-1962) ; cf. Général Maurice Faivre, Les combattants musulmans de la guerre d'Algérie, Paris, L'Harmattan, 1995.

58. C'est-à-dire « [à] ceux qui, épris d'absolu, en définissent le sens » (Camus).

59. Armée de Libération Nationale (1954-1962).

60. Armée Nationale Populaire (depuis 1962).

61. Précisément parce qu'elle s'inscrit en faux contre le mythe de l'unanimité des masses derrière le F.L.N.

62. Nous n'avons certes pas les moyens empiriques d'être définitif sur le statut du harki dans les représentations des gens ordinaires en Algérie. Il faudrait, pour ce faire, que soient réunies les conditions sociales et politiques (tant en termes de pacification des esprits que de garantie des libertés publiques) hors lesquelles toute recherche de terrain (notamment sous la forme d'entretiens approfondis menés auprès d'un échantillon représentatif de la population algérienne) se heurterait à des entraves matérielles et symboliques rédhibitoires. Cependant, l'hypothèse de la fonction « conjuratoire » de la figure du harki dans l'ordinaire des prises de position se fonde sur un faisceau d'indices redevables tant des conversations informelles que nous avons pu mener avec des personnes issues de l'immigration algérienne que de la campagne d'entretiens approfondis que nous avons menés en France auprès de plusieurs dizaines de fils et filles de harkis. Ceux-ci, évoquant la nature de leurs interactions quotidiennes avec certains enfants d'immigrés algériens préalablement informés de leur appartenance à la communauté harkie, nous ont fait part des tensions (voire des altercations) que ce type de face-à-face était susceptible de provoquer. La stigmatisation des enfants de harkis par certains jeunes « beurs » peut être interprétée comme une tentative de retournement du stigmate et de réappropriation symbolique du roman familial. L'algérianité originelle et la trajectoire dessinée par la migration socio-économique des parents, ordinairement vécues comme des facteurs de fragilisation identitaire, peuvent être extra-ordinairement valorisées par contraste avec la situation des anciens harkis et de leurs enfants, censés avoir trahi leurs origines pour se faire une place dans la société d'accueil. D'autre part, plusieurs des enfants de harkis que nous avons interrogés nous ont fait part de la prégnance de l'acception générique du terme harki (usitée pour fustiger les personnes dont le comportement est censé attenter aux intérêts de la collectivité) dans le vocabulaire routinier des 
Algériens de France et d'Algérie. Alors qu'elle visionnait, via le satellite, un programme sportif retransmis par la télévision algérienne, la fille d'un ancien supplétif de l'armée française fut ainsi stupéfaite d'entendre le commentateur qualifier de « harki » un footballeur qui avait marqué contre son camp. Cependant, ces formes routinières de stigmatisation s'apparentent davantage à des mécanismes conventionnels de " réassurance identitaire » (se « re-définir » par contraste avec l'autre), régis selon une trame stéréotypée héritée pour partie des formes orchestrées de la stigmatisation. Elles ne présagent en rien de ce que pensent en leur for intérieur les Algériens de France et d'Algérie. Le contexte, dans le bled comme dans certaines cités, se prête moins à l'interrogation des faits qu'à leur conjuration.

63. "Algérie : les grands cimeterres sous la lune », entretien conduit par Amir Taheri auprès d'un officier algérien de haut rang sous couvert d'anonymat, Politique internationale, printemps 1998.

64. Ce que la loi sur la « concorde civile » dépeint comme la voie d'une possible " réinsertion civile au sein de la société » (Dispositions générales, article 1er).

65. Les « patriotes » sont des villageois armés par les autorités pour se constituer en autodéfense dans les zones non « sécurisées " par l'armée (si tant est que l'on puisse dire que l'A.N.P. fasse œuvre de sécurisation en Algérie depuis l'interruption du processus électoral de 1992).

66. Florence Aubenas, "A Jijel, le temps de la réconciliation », Libération, mardi 14 septembre 1999, p. 12.

67. « La communauté nationale, ici et ailleurs, se doit d'assumer ses enfants, tous ses enfants. Cette erreur, au demeurant tragique, a été commise à l'indépendance de l'Algérie. Elle ne sera pas commise avec moi. A l'indépendance, nous avons traité le problème des harkis de façon collective. Nous sommes en train de payer la facture. Une partie des maquis, ce sont des enfants de harkis » (propos repris in El Watan, mardi 14 septembre 1999, p. 4). On peut lire en filigrane des déclarations d'Abdelaziz Bouteflika une reconnaissance à peine voilée, quoique livrée sur un mode euphémique, tant des massacres qui ont suivi l'indépendance de l'Algérie que de l'implication des autorités algériennes d'alors; néanmoins, au passage, l'assimilation des enfants de harkis aux maquisards islamistes est réitérée, quoique plus subtilement puisque non sur un mode uniment accusateur mais sur un mode partiellement auto-accusateur : certes, les enfants de harkis, tout comme leurs pères, sont des « monstres », mais c'est nous qui les avons engendrés.

68. Mouvement Algérien des Officiers Libres.

69. Communiqué du mardi 11 janvier 2000.

70. Cf. « Le serment » du M.A.O.L. ; ce texte, ainsi que ceux dont sont extraits les citations à suivre, est consultable dans son intégralité sur le site internet du M.A.O.L. : http://www.anp.org.

71. Cf. «Le nouvel appel du 1er novembre».

72. Cf. «L'Armée Nationale Populaire : Vérités».

73. Selon Mohamed Benrabah, « le régime n'a jamais cessé de violenter la société algérienne en lui imposant l'enfermement contre sa nature plurielle. (...) En renforçant les seuls caractères arabe et islamique de l'identité nationale, on nie les racines berbéro-latino-judéo-hispano-turco-franco-algériennes. (...) Pour faire "plus Arabe que les Arabes", on inculque le mépris des ancêtres berbères et on oblige les enfants à passer le plus clair de leur temps à faire l'histoire des autres : celle du Moyen-Orient. [Par surcroît], on leur transmet une histoire glorifiant la violence » (Mohamed 
Benrabah, «Bouteflika arrachera-t-il l'Algérie à son enfermement? », Libération, lundi 13 septembre 1999, p. 6)

74. Le mythe de l'unanimité des masses derrière le F.L.N. depuis le 1er novembre 1954 jusqu'à l'indépendance, soit le mythe d'une nation « conscientisée » dans et par la praxis révolutionnaire, est le dernier des grands mythes fondateurs à même de justifier la prédominance du militaire sur le politique en Algérie. C'est pourquoi il importe aux autorités d'occulter l'historicité réelle du rapport des forces au sein de cette part de la population algérienne - c'est-à-dire la population musulmane - dont le F.L.N. revendiquait l'exclusivité de la représentation. Or, la grande majorité de la population musulmane est restée attentiste au moins jusqu'au moment où il est apparu clairement que le général de Gaulle engageait inexorablement l'Algérie française dans la voie de la décolonisation

75. Daniel Sibony, op. cit.

76. Jacques Le Goff, Histoire et mémoire, Paris, Gallimard, 1988, cité par Fouad Soufi, "Pratiques historiographiques et mythes de fondation. Le cas de la Guerre de libération à travers les institutions d'éducation et de recherche algériennes » in Charles Ageron, op. cit, pp. 305-322.

77. Comme étant une autre figure $\mathrm{du}$ « nous ».

78. L'une des principales revendications de groupements tels que le collectif Justice pour les harkis ou l'association Jeune Pied-Noir.

79. De simples déclarations d'intentions, qui tiennent sur vingt-quatre lignes, intertitres compris.

80. Guy Pervillé, « Les accords d'Evian et les relations franco-algériennes » in J.-P. Rioux (dir.), La guerre d'Algérie et les Français, Paris, Fayard, 1990, pp. 487-489. C'est nous qui soulignons.

81. Les « opérations de police » étant la composante même du « bourbier » algérien.

82. Guy Pervillé, op. cit., pp. 487-489 et 492-493.

83. Cette expression renvoie à celle d' « intérêts stratégiques ", l'emploi de l'adjectif « vital » ne devant, à cet égard, pas prêter à confusion : il ne s'agit pas, la plupart du temps, d'y inclure la préservation des vies humaines...

84. Cf. Emmanuel Brillet, « De la congruence entre l'intellection et l'intervention: Sartre et Camus en guerre d'Algérie ", in Cahiers Politiques, n ${ }^{\circ}$, juin 2000, pp. 31-58.

INDEX

Index géographique : Algérie, France, Maghreb

Mots-clés : victimes et victimisation, massacre de masse, Théorie politique 